\title{
Size-Induced Reduction of Transition Pressure and Enhancement of Bulk Modulus of AIN Nanocrystals
}

\author{
Zhongwu Wang,* Kimberley Tait, Yusheng Zhao, and David Schiferl \\ Los Alamos National Laboratory, Los Alamos, New Mexico 87545 \\ Changsheng Zha \\ CHESS, Wilson Laboratory, Cornell University, Ithaca, New York 14853
}

Hinako Uchida and Robert. T. Downs

Department of Geosciences, University of Arizona, Tucson, Arizona 85721

Received: April 12, 2004; In Final Form: May 26, 2004

\begin{abstract}
An in situ X-ray-diffraction study of AlN nanocrystals under hydrostatic (or quasihydrostatic) conditions was performed to pressures of $36.9 \mathrm{GPa}$, using an energy dispersive synchrotron-radiation technique in a diamondanvil cell (DAC). Hexagonal AIN nanocrystals have a particle size of $10 \mathrm{~nm}$ on average, and display an apparent volumetric expansion as compared to the bulk AlN polycrystals. Upon compression to $14.5 \mathrm{GPa}$, AlN nanocrystals start to transform to a rocksalt structure phase. This pressure is significantly lower than the transition pressure of $22.9 \mathrm{GPa}$ observed from the bulk AlN by using the same technique. The nanosized wurtzite phase has a bulk modulus $\left(B_{0}\right)$ of $321 \pm 19 \mathrm{GPa}$, larger than that of the bulk AlN crystals with a $B_{0}$ of $208 \mathrm{GPa}$. There is a large volumetric decrease of $20.5 \%$ upon the wurtzite-to-rocksalt phase transformation. Combination of the size-induced volumetric expansion and resulting softening of the Poisson ratio and shear modulus may explain the reduction of transition pressure in this type of nanomaterials.
\end{abstract}

Because of the relevance to the engineering of materials with enhanced mechanical properties, nanocrystals have been of considerable interest for current studies in terms of pressure tuning. Previous studies indicate that, as for a large number of materials, decrease of particle size results in an enhancement of elastic modulus, ${ }^{1-4}$ but their pressure-induced phase transformations exhibit the two reversal trends: the reduction verses the elevation of the phase transition pressure. ${ }^{2-4}$ Enhancement of the bulk modulus and of transition pressure was previously explained by an increased surface energy contribution, ${ }^{2}$ but the mechanism resulting in the reduction of transition pressure still remains unclear. ${ }^{3,4}$ However, it is observed that the studied nanocrystalline materials that display a weakened transition pressure have the particle size below $15 \mathrm{~nm}, 3,4$ and this is coincidently smaller than the critical size of $15 \mathrm{~nm}$ that was observed from numerous nanocrystals. ${ }^{5-8}$ Below the critical size, nanocrystals exhibit several reverse properties, such as the volumetric expansion and softening of the Poisson ratio and shear modulus. ${ }^{5-7}$ These are different from the nanosize induced volumetric contraction and the enhanced shear modulus. Therefore, we expect that the nanocrystals with a particle size smaller than the critical size may display a reduction of transition pressure for the pressure-induced first-order solid-solid phase transformation. To explore the reliability of such an assumption and any potentially novel mechanism for the ultrafine nanocrystals $(<15 \mathrm{~nm})$, AlN nanocrystals with an average particle size of $10 \mathrm{~nm}$ were studied by using a Diamond Anvil Cell (DAC) coupled with the high-energy synchrotron X-ray diffraction to $36.9 \mathrm{GPa}$. The results demonstrate that AlN nanoc-

* Author to whom correspondence should be addressed. Phone: 505 667-8839. Fax: 505-665-2676. Email: z_wang@lanl.gov.

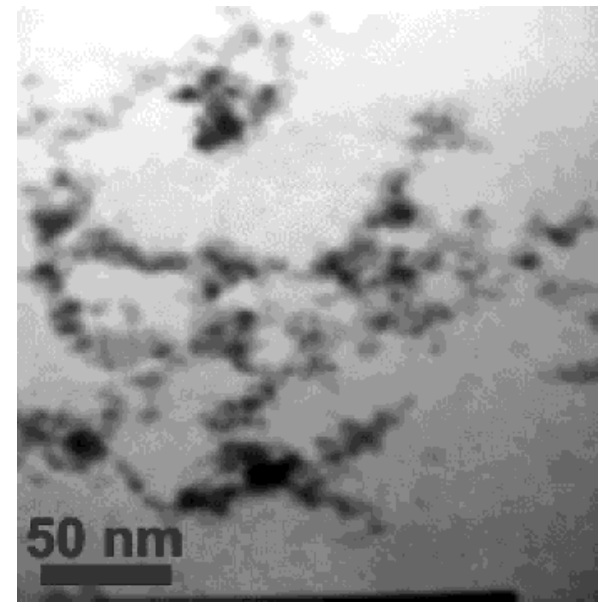

Figure 1. The transmission electronic microscopy (TEM) image of AlN nanocrystals.

rystals exhibit a significant reduction of transition pressure for the wurtzite-to-rocksalt phase transformation, and still display an enhanced bulk modulus.

A sample of AlN used in this study has a nearly spherical particle size of $10( \pm 2) \mathrm{nm}$, on average (Figure 1). X-ray diffraction characterized that AlN nanocrystals have a hexagonal wurtzite structure. High-pressure X-ray diffraction measurements were performed at room temperature by using a gasketed highpressure Diamond Anvil Cell (DAC). ${ }^{1,4}$ A mixture of $~ 97 \%$ AlN nanopolycrystals and $\sim 3 \%$ platinum with the liquid pressure transmission medium (methanol/ethanol 4:1) was loaded in a T301 steeliness gasket hole that is $80 \mu \mathrm{m}$ in initial thickness and $150 \mu \mathrm{m}$ in diameter. The liquid pressure medium 


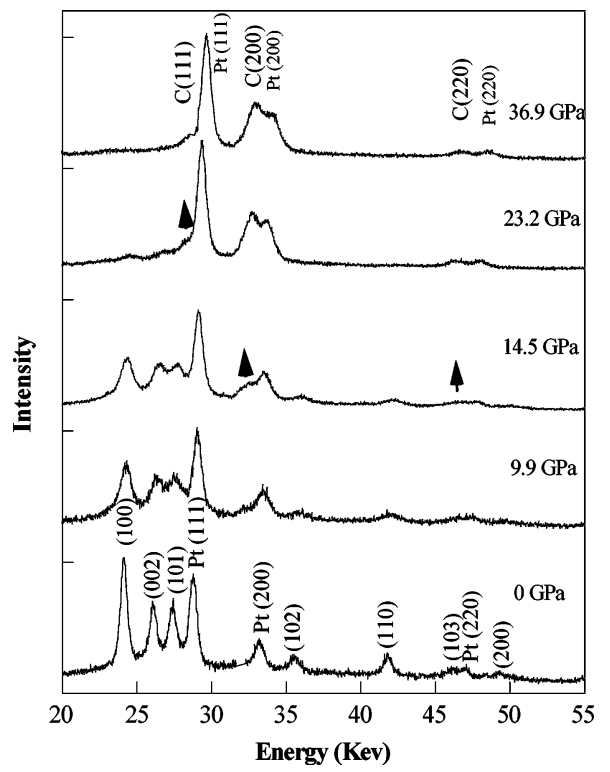

Figure 2. High-pressure X-ray-diffraction spectra of AlN nanocrystals. Note: The upward arrows ( $\uparrow$ ) denote the occurrence of the new peaks of the cubic rocksalt AlN phase.

is used to maintain the sample having a hydrostatic or quasihydrostatic state under pressure, to circumvent the interaction between the particles and possibly resulting enlargement of the particle size, as well as the generation of the large pressure gradient in the sample. Platinum serves to read the pressure at each X-ray diffraction collection. Energy dispersive synchrotron radiation at CHESS was used for X-ray diffraction measurements. ${ }^{1,4}$ Energy calibrations were made by using well-known radiation sources $\left({ }^{55} \mathrm{Fe}\right.$ and $\left.{ }^{133} \mathrm{Ba}\right)$, and angle calibrations at a fixed angle of $11^{\circ}(2 \theta)$ were made from the six peaks of standard Au powder. Powder X-ray diffraction patterns were collected to pressures of $\sim 36.9 \mathrm{GPa}$ for the determination of structure and refinement of cell parameters.

Figure 2 shows X-ray diffraction patterns of AlN nanocrystals to pressures of $36.9 \mathrm{GPa}$. The unit cell parameters are calculated from the X-ray diffraction peaks of the starting sample at $1 \mathrm{~atm}$ of pressure, which show a pure hexagonal wurtzite phase with $a_{0}=3.1203(9) \AA, c_{0}=4.9921(12) \AA$, and $V_{0}=42.09(8) \AA^{3}$. As compared to the reported unit cell parameters of the AlN macrocrystals with $a_{0}=3.114 \AA, c_{0}=4.9792 \AA$, and $V_{0}=$ $41.74 \AA^{3},{ }^{9}$ it is clear that both the $a$ - and $c$-axis exhibit an apparent enlargement, and that the enlargement of the $c$-axis is slightly greater than that of the $a$-axis. The unit cell volume is $0.8 \%$ larger than that of the bulk AIN crystal. This confirms that the size-induced volumetric expansion apparently occurs in such ultrafine nanocrystals, and differs from the volumetric contraction observed from the large numbers of nanocrystalline materials that have the particle size larger than the critical size of $15 \mathrm{~nm}$.

Upon elevation of pressure to $14.5 \mathrm{GPa}$, several new peaks of the high-pressure phase started to emerge. This pressure is drastically lower than the phase transition pressures of 22.9 and $21 \mathrm{GPa}$, which were observed from the bulk AlN polycrystals by using the same technique and the shock wave impact technique, respectively. ${ }^{10-12}$ Previous studies have demonstrated that the cold compression DAC data are quite compatible with the shock wave-impacted data, so the comparison between this study and the above two types of high-pressure results is reliable. But, one pressure study performed at in situ pressure heating conditions produces a quite low transition pressure of 16.5 $\mathrm{GPa}^{12} \mathrm{As}$ is well-known, in situ heating can significantly

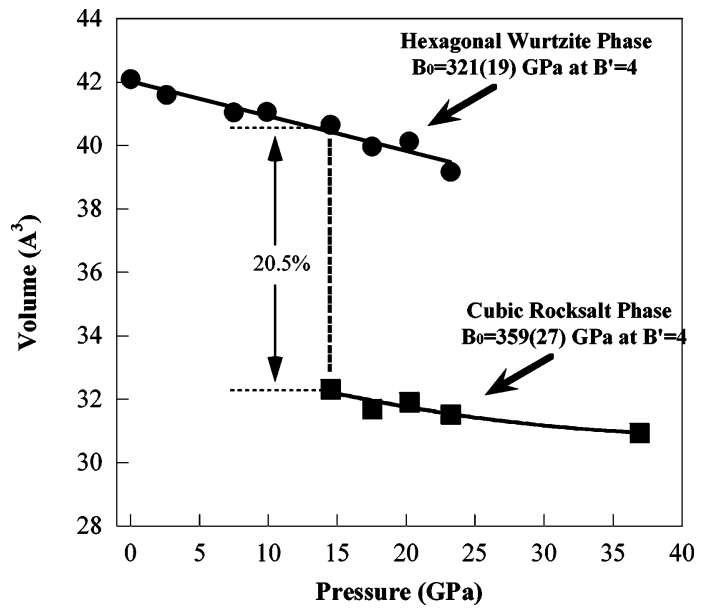

Figure 3. Equation of state (EOS) curve and the relative volumetric variation of AlN upon the phase transformation at $14.5 \mathrm{GPa}$. Note: For better comparison of the volumetric variation between the two phases, the volumes of the wurtzite and rocksalt phases are shown by the same units of $2 \mathrm{AlN}$, which corresponds to one wurtzite unit cell $(M=2)$ and a half rocksalt unit cell $(M=4)$. $M$ denotes the unit number of $\mathrm{AlN}$ in one unit cell.

overcome the existing energy gap upon the phase transformation, and correspondingly lead to the occurrence of the lower transition pressure and further speed up the accomplishment of the phase transformation, so it is reasonable to observe a lower transition pressure at in situ heating conditions relative to that under cold compression. ${ }^{10,12}$ Moreover, we have to consider the effect of the pressure-induced nonhydrostaticity in the sample, because the large pressure gradient can result in a significant decrease of transition pressure. In this study, we use the liquid pressure transmission medium to maintain a hydrostatic condition, and this type of pressure medium (M:E 4:1) is able to maintain a hydrostatic state up to $15 \mathrm{GPa}$, and above $15 \mathrm{GPa}$, the solidification of the liquid medium leads to the occurrence of the quasihydrostaticity in the sample chamber. Because the observed transition pressure of $14.5 \mathrm{GPa}$ is located within the hydrostatic pressure range, it is thus believed that such a reduced transition pressure is not caused by the large pressure gradient across the sample. As a result, it is guaranteed that the observed transition pressure is truly lower than that of the bulk AIN crystals.

The high-pressure phase of AlN was indexed to the cubic rocksalt structure with the unit cell parameters of $a=4.0133$ $\AA$ and $V=64.64 \AA^{3}$ and four units of AlN per unit cell at a pressure of $14.5 \mathrm{GPa}$. The bulk modulus of the rocksalt phase was calculated to be $359(27) \mathrm{GPa}$ at a fixed $B_{0}{ }^{\prime}=4$, which is slightly higher than the $B_{0}$ of 285-329 GPa obtained from the bulk AlN polycrystals. ${ }^{13}$ At the phase transition pressure of 14.5 $\mathrm{GPa}$, the high-pressure rocksalt phase is $20.5 \%$ denser than the hexagonal wurtzite phase (Figure 3). Upon release of pressure, the rocksalt phase was preserved at 1-atm pressure conditions. Previous study indicates that the high-pressure rocksalt phase is not quenchable upon decompression to zero pressure in the case of no in situ heating under pressure. ${ }^{10}$ However, it should be pointed out that the peak pressure in that study was reached only to $30 \mathrm{GPa}$. Thus, combining previous results with this study that reached the peak pressure of $36.9 \mathrm{GPa}$, it is reasonably suggested that a critical pressure representing the completion of the phase transition is around $35 \mathrm{GPa}$. At ambient conditions, the cell parameters of the rocksalt phase were calculated to be $a_{0}=4.0445(3) \AA$ and $V_{0}=66.16 \AA^{3}$, which has no significant difference from that obtained from in situ high-temperature high- 
TABLE 1: Parameters for the Transition and Equation of State (EOS) Data of the Wurtzite-to-Rocksalt Phase Transformation

\begin{tabular}{|c|c|c|c|c|c|c|}
\hline method & $\begin{array}{l}\text { particle } \\
\text { size }\end{array}$ & $\begin{array}{c}\text { transition } \\
\text { pressure } \\
(\mathrm{GPa})\end{array}$ & $\begin{array}{l}\Delta V \\
(\%)\end{array}$ & $\begin{array}{c}B_{0} \\
(\mathrm{GPa})\end{array}$ & $B^{\prime}$ & \\
\hline diffurotio & bulk & 22.9 & 18.0 & 208 & 6.3 & \\
\hline $1600^{\circ} \mathrm{C}$ & bulk & 16.5 & 20.6 & & & \\
\hline shock wave & bulk & 21.0 & 22.0 & & & \\
\hline X-ray diffraction & nanosize & 14.5 & 20.5 & 321 & 4.0 & \\
\hline
\end{tabular}

${ }^{a}$ This study.

pressure conditions. ${ }^{12}$ This is likely caused by a pressure-induced increase of particle size. ${ }^{14}$

Figure 3 shows the equation of state (EOS) data of AlN nanocrystals to pressures of $36.9 \mathrm{GPa}$. A fit to the BirchMurnaghan EOS of the hexagonal wurtzite phase gives the bulk modulus $B_{0}=321(19) \mathrm{GPa}$ at a fixed $B_{0}{ }^{\prime}=4$. This is significantly greater than the $B_{0}$ of $208 \mathrm{GPa}$ observed from the bulk AlN crystals (Table 1). ${ }^{10-12}$ Therefore, this indicates that the reduction of particle size can significantly lead to an enhancement of the bulk modulus. This is consistent with the previous studies on a significant ratio of nanocrystals. ${ }^{1-4}$

Numerous studies on nanosized materials indicate that the decrease of particle size leads to an apparent elevation of phase transition pressure and of bulk modulus as compared to their bulk counterparts. ${ }^{1,2}$ But, recent studies on $\gamma-\mathrm{Fe}_{2} \mathrm{O}_{3}$ and $\mathrm{CeO}_{2}$ nanocrystals reveal that the decrease of particle size results in a significant reduction of the phase transformation pressure for the pressure-induced first-order solid-solid-phase transformation..$^{3,4}$ The enhancement of bulk modulus can easily be explained by a higher surface energy contribution, but the mechanism resulting in a reverse variation of phase transition pressure remains unclear. Moreover, at 1-atm pressure conditions, studies uncovered the existence of a critical size for a large number of nanocrystalline materials, and such a critical size was observed mostly to be around $15 \mathrm{~nm} .{ }^{5-7}$ Above the critical size, nanocrystals typically exhibit an apparent volumetric contraction, but below the critical size, they display a reverse volumetric expansion together with a noticeable softening of the Poisson ratio and of shear modulus..$^{5-8}$ It is also noticed that, as for these nanocrystals (e.g. $\gamma-\mathrm{Fe}_{2} \mathrm{O}_{3}$ and $\mathrm{CeO}_{2}$ ) that exhibit a reduced transition pressure in the pressure-induced first-order solid-solid-phase transformation, the particle sizes of the nanocrystals studied previously are coincidently smaller than the critical size of $15 \mathrm{~nm} .^{3,4}$ Thus, it is logically suggested that the combination of the size-induced volume expansion and softening of the Poisson ratio and shear modulus may play a crucial role in the reduction of the transition pressure for the first-order solid-solid-phase transformation in these nanomaterials, such as $\gamma-\mathrm{Fe}_{2} \mathrm{O}_{3}, \mathrm{AlN}$, and $\mathrm{CeO}_{2}$. Since these nanomaterials continue to exhibit an enhanced bulk modulus, while the particle size is smaller than the critical size, it is implied that the higher surface energy contribution still puts a considerable impact on the enhanced bulk modulus. It must be pointed out that CdSe nanocrystals with a particle size of $4.4 \mathrm{~nm}$ do not exhibit a reduced transition pressure, and instead an increased transition pressure was observed. ${ }^{2}$ However, recent study indicates that $\mathrm{CdSe}$ nanocrystals with a particle size ranging from 2.5 to $6 \mathrm{~nm}$ unexpectedly display an apparent volumetric contraction, ${ }^{15}$ rather than an expansion. Thus, this allows us to conclude that the reduction of phase transition pressure observed in these ultrafine nanocrystals (e.g. $\gamma-\mathrm{Fe}_{2} \mathrm{O}_{3}, \mathrm{AlN}$, and $\mathrm{CeO}_{2}$ ) results from the size-induced volumetric expansion and the softening of both the Poisson ratio and shear modulus.

In summary, we have investigated the AlN nanocrystals to $36.9 \mathrm{GPa}$ at room temperature and at a hydrostatic or quasihydrostatic condition by using high-energy synchrotron X-ray diffraction. AlN nanocrystals have an average particle size of $10 \mathrm{~nm}$ and exhibit a noticeable volumetric expansion, which is $0.8 \%$ larger than the bulk AlN. The wurtzite AlN nanocrystals initially transform to the rocksalt structure at $14.5 \mathrm{GPa}$, which is significantly lower than that observed from the bulk AlN polycrystals. The bulk modulus of the wurtzite phase is 321(19) GPa at a fixed $B_{0}=4$, which is notably greater than that of the bulk AlN of $208 \mathrm{GPa}$. It is suggested that the combination of the volume expansion and the softening of the Poisson ratio and shear modulus results in the reduction of the phase transition pressure, and that a higher surface energy contribution still casts a crucial effect on the enhanced bulk modulus of these types of nanomaterials.

Acknowledgment. The Director's Postdoctoral Fellowship Fund at Los Alamos National Laboratory (LANL) supported this study. Part of the work was conducted at Cornell High Energy Synchrotron Source (CHESS), which is supported by NSF and NIH/NIGMS under award DMR 9713424.

\section{References and Notes}

(1) Wang, Z. W.; Zhao, Y.; Schiferl, D.; Qian, J.; Downs, R. T.; Mao, H. K.; Sekine, T. J. Phys. Chem. B 2003, 107, 14151.

(2) Tolbert, S. T.; Alivisatos, A. P. Science 1994, 265, 373.

(3) Jiang, J. Z.; Olsen, J. S.; Gerward, L.; Morup, S. Europhys. Lett. 1998, 44, 620.

(4) Wang, Z. W.; Saxena, S. K.; Pischedda, V.; Liermann, H. P.; Zha, C. S. Phys. Rev. B 2001, 64, 012102.

(5) Villain, P.; Goudeau, P.; Renault, P. O.; Badawi, K. F. Appl. Phys. Lett. 2002, 81, 4365 .

(6) Shen, T. D.; Koch, C. C.; Tsui, T. Y.; Pharr, G. M. J. Mater. Res. 1995, 10, 2892. 825 .

(7) Zhou, Y.; Erb, U.; Aust, K. T.; Palumbo, G. Scr. Mater. 2003, 48,

(8) Spanier, J. E.; Robinson, R. D.; Zhang, F.; Chan, S. W.; Herman, I. P. Phys. Rev. B 2001, 64, 245407.

(9) Powder diffraction file: PDF No. 25-1113 with $a_{0}=3.1114 \AA, c_{0}$ $=4.9792 \AA$, and $V_{0}=41.74 \AA^{3}$.

(10) Ueno, M.; Onodera, A.; Shimomura, O.; Takemura, K. Phys. Rev. $B$ 1992, 45, 10123 .

(11) Kondo, K.; Sawaoka, A.; Sato, K.; Ando, M. In Proceedings of the Conference on Shock Waves in Condensed Matter; Nellis, W. J., Ed.; AIP Conf. Proc. No. 78; AIP: New York, 1981; p 325.

(12) Vollstädt, H.; Ito, E.; Akimoto, M.; Fukunaga, O. Proc. Jpn. Acad. Ser. $B$ 1990, 66, 7 .

(13) Uehara, S.; Masamoto, T.; Onodera, A.; Ueno, M.; Shimomura, O.; Takemura, K. J. Phys. Chem. Solids 1997, 58, 2093.

(14) Wang, Z. W.; Zhao, Y.; Schiferl, D.; Zha, C. S.; Downs, R. T. Appl. Phys. Lett. 2004, 85, 124.

(15) Zhang, J. Y.; Wang, X. Y.; Xiao, M.; Qu, L.; Peng, X. Appl. Phys. Lett. 2002, 81, 2076. 